\title{
Thorax
}

Editorials

\section{Health service accessibility and deaths from asthma}

\author{
J E Garrett
}

The finding of Jones and Bentham ${ }^{1}$ in this issue of Thorax is of importance since it is the first time geographical barriers to acute hospital services has been defined as a risk factor for asthma mortality. Asthma mortality rates can vary by two to threefold between countries and even between regions within countries. In New Zealand, for example, at the height of the epidemic of asthma deaths in the late 1970 s and early 1980 s there were 2.5 -fold differences in crude mortality rates between districts $(2.7-6.9 / 100000){ }^{2}$ Generally, mortality rates were highest in those areas where there was low population density and large distances to secondary health services, though this was not consistently observed. Presumably such variations are a consequence of the combined effects of cultural, environmental, and socioeconomic forces on risk factors for asthma mortality, which may include distance from hospital. Aware of such variations, and in acknowledgement that they could not control for a number of known risk factors for asthma mortality (such as a previous life threatening attack, need for continuous prednisone, discontinuity of care, and psychosocial factors), ${ }^{3}$ Jones and Bentham modelled for inter-regional variance in mortality rates in their analysis. As a consequence it could be argued that they "controlled" for a number of potential confounders by the method of analysis employed. This may be important since there is imprecision in the measurement of the potential confounding factors for which they were able to control namely, socioeconomic status, social isolation, ownership of a car, and population density. At the completion of their analysis they noted a small but significant association between increasing distance from an acute hospital and asthma mortality. Because of the linearity of the relationship (which reinforces the likelihood that the association is real) and the potential importance of the finding, this paper warrants further discussion.

Observations of geographical and temporal variations in health outcomes are one of the foundations of epidemiological analysis. Before undertaking further research on the subject it would be important to obtain longitudinal data to explore the relationship between distance from acute hospital services and asthma mortality over time to help ensure that the preliminary findings are not artifactual. Promoting equity in utilisation of medical services has become a policy goal for the health services in many countries including the United Kingdom. As such, one would hope that improved quality of health care in association with improved emergency services within the community $^{4}$ (for example, after hours medical care and ambulance services) would weaken the relationship between geographical isolation and asthma mortality over time. If this were not shown to be true then the finding of
Jones and Bentham would be of particular importance to public health authorities.

The data available to Jones and Bentham were population based rather than having been gained specifically from individuals with asthma. This not only limits the accuracy of their study, but also means we can only speculate as to the reason for the association. It is generally acknowledged, however, that rapid access to medical care and good quality care (both of the acute attack and of long term management) are of critical importance in preventing mortality from asthma. ${ }^{5}$ As such, one of the studies that would need to be undertaken is a comparison of the quality of asthma management in communities remote from hospital services with those in close proximity. It would be important to collect the information in such a manner as to be able to interpret any differences observed. It may be difficult, for example, to determine what impact hospital services such as outpatient clinics and emergency departments have on asthma morbidity and mortality rates.

Whilst hospital based care of acute asthma could be criticised because it is often poorly timed and encourages discontinuity of care, it can be shown to have positive effects in addition to the obvious one of preventing asthma deaths. New interventions in asthma management are more usually initiated by hospital based specialists and these subsequently "filter" out into the community. Patients living in close proximity to hospitals therefore gain access to these interventions before those living distant, not only as a consequence of an increased rate of hospital admission, ${ }^{6}$ but also because of subsequent referral to specialist asthma clinics. ${ }^{7}$ As a consequence, any study undertaken to examine differences in management practices between urban and remote rural communities would be expected to define differences in self-management skills in individual asthmatic patients as well as in the frequency of prescription and dose of inhaled steroids. There would be a dilemma as to how to interpret these differences which may not necessarily explain why those living distant from acute hospitals are at increased risk of dying. Further, if one were to try to rectify any differences in care one would be unsure whether to improve asthma management by general practitioners living in remote districts or to provide ambulatory specialist asthma clinics to remote areas.

Any prospective study would also need to take into account the pattern of utilisation of health services, particularly acute services. Like asthma deaths, hospital admissions are a consequence of a complex relationship between disease severity, illness behaviour, and utilisation of health care services. ${ }^{8}$ For example, we have found that patients who live in close proximity to an acute district hospital are three times more likely to use the emergency department and to be admitted than those living distant. ${ }^{6}$ 
Strachan et $a l^{9}$ described substantial increases in hospital admissions in England during the 1980s which seemed to be a result of changes in provision and utilisation of health services, particularly after hours, rather than a consequence of changes in asthma prevalence or severity. There are a number of potential barriers to primary health care which may influence illness behaviour and thus the utilisation of health care services:

1. Financial. Whilst the direct cost of a general practitioner visit is not a particular problem in England and Wales, part charges on asthma therapy are a potential barrier and the indirect costs of obtaining medical care can be problematical, particularly for the poor.

2. Organisational. Poor organisation of community health services after hours would be expected to have a greater impact on patients living remote from hospital services than on those living in close proximity.

3. Geographical. Asthmatic patients who live in remote communities may need to travel large distances to see a general practitioner, particularly after hours. ${ }^{10}$

4. Attitude. Asthmatic patients who live in rural communities appear to use general practitioners less. ${ }^{11}$ Particularly in small communities which are less mobile, strong relationships may develop between doctors and their patients which may reduce a patient's willingness to access a different doctor who is providing the after hours service. Patients who have previously been admitted to hospital may decide that hospital rather than community based care is more appropriate and there may be perverse financial incentives which may prompt general practitioners to encourage patients to access hospitals for management of acute asthma.

5. Medications. A number of papers have shown a relationship between the amount of $\beta$ agonist inhaled and the severity of an asthma attack. ${ }^{12-14}$ To date no explanation for this association has been proven, but possibilities include (a) increased $\beta$ agonist usage reflects severity of the attack or downregulation of $\beta$ agonist receptors, (b) inhaled $\beta$ agonists in increasing dose worsen the severity of the attack, or (c) inhaled $\beta$ agonists may simply delay patients seeking medical attention earlier (the most likely explanation). As a consequence, the prescription of nebulised $\beta$ agonists to patients living in remote districts should be discouraged unless patients have a clear management plan, including when and how to access medical care if their attack is failing to respond to nebulised therapy. They should also have exhibited appropriate behaviour previously in response to worsening asthma.

Patients who live distant from hospital services seem to use the emergency department earlier in the course of their attack than those living in close proximity, ${ }^{6}$ so they do seem to access health services earlier. Furthermore, patients living distant from hospitals are more likely to be admitted to hospital following referral by a general practitioner than those living nearby who more usually self refer, again reflecting their different pattern of health care utilisation. ${ }^{6}$ A delay in seeking medical attention would be expected to have more severe repercussions for patients living in remote districts, and this remains the most biologically plausible explanation for the findings reported by Jones and Bentham. It would also explain the linear relationship between increasing distance from acute hospital services and death from asthma.

Whilst acknowledging that the association between geographical distance from acute hospital services and mortality was relatively weak, there are a number of factors in the analysis which may have reduced the chances of showing a significant relationship. For example, straight line distance to the nearest hospital is a less accurate method of defining access to hospital services than the actual time it takes to get to hospital. Ideally, risk factors associated with asthma mortality should have been controlled for but were not available to the authors. However, by modelling for inter-regional variances and then controlling for possible confounding factors they may have adjusted for some factors which were intermediate in the causal pathway and which would have reduced the likelihood of showing an association. On the other hand, one cannot discount the possibility that the relationship between distance from hospital and asthma mortality is indeed confounded. It is possible, for example, that asthmatic subjects with important psychosocial problems or who have more severe asthma are more likely to live in remote districts, perhaps to escape the pressures of residing in a high density population or a polluted urban environment. However, this seems unlikely. Jones and Bentham should therefore be congratulated on performing this preliminary study which should be perceived as hypothesis generating. Hopefully, however, it will stimulate further research - both in the United Kingdom and internationally.

Green Lane Hospital,

J E GARRETT

Auckland 3,

New Zealand

1 Jones AP, Bentham G. Health service accessibility and deaths from asthma in 401 local authority districts in England and Wales, 1988-92. Thorax in 401 197; 52:218-22.

2 Sears MR, Rea HH, Beaglehole R, Gillies AJ, Holst P, O'Donnell TB, et al. Asthma morbidity in New Zealand: a 2 year national study. NZ Med al. Asthma morbidit

3 Rea HH, Scraggs R, Jackson R, Beaglehole R, Fenwick J, Sutherland DC. A case-control study of deaths from asthma. Thorax 1986;41:833-9.

4 Capewell S. The continuing rise in emergency admissions. BMF 1996;312 991-2.

5 Fitzgerald M, Macklem PT. Proceedings of a workshop on near fatal asthma. Can Respir F 1995;2:113-26.

6 Garrett J, Mulder J, Wong-Toi H. Characteristics of asthmatics using an urban accident and emergency department. NZ Med f 1986;101:359-61. McClellan VE, Garrett J. Attendance failure at Middlemore Hospital asthma clinic. NZ Med f 1989;102:211-3.

8 Anderson HR. The epidemiological value of hospital diagnostic data. In: Bennett AE, ed. Recent advances in community medicine. Edinburgh Churchill Livingstone 1978: 175-94.

9 Strachan DP, Anderson HR. Trends in hospital admission rates for asthma in children. BMF 1992;304:819-20.

10 Parkin D. Distance as an influence on demand in general practice. $\mathscr{f}$ Epidemiol Community Health 1979;33:96-9.

11 Taytard A, Tessier JF, Gervais M, Gachie JP, Douet C, Kombou L, et al. Actual usage of medical facilities by asthmatics in two French rura settings: a preliminary study. Eur Respir f 1990;3:856-60.

12 Garrett J, Frankel A, Lanes S, Rodwell P, Kelly AM, Town GI. Factors predicting severity and response to therapy in acute asthma. Am $\mathcal{F}$ Respir Crit Care Med 1995;151:A380.

13 Spitzer WO, Suissa S, Ernst P, Horwitz P, Hubbick B, Cockroft D, et al. The use of $\beta$ agonist and risk of death and near death from asthma. $N$ Engl $\mathcal{F}$ Med 1992;326:501-6.

14 Abramson M, Driver J, Willis J, Evans N, McNeill J. Blood salbutamol levels are higher in asthma deaths than controls. Aust NZ F Med 1997 (in press). 